\title{
Support for Eddington's Number and His Approach to Astronomy: Recent Developments in the Physics and Chemistry of the Human Brain
}

\author{
Michael A. Persinger \\ Laurentian University, Sudbury, Ontario, P3E2C6, Canada \\ E-mail address: mpersinger@laurentian.ca
}

\begin{abstract}
The astronomical contributions of Sir Arthur Eddington have sometimes been distracted by his philosophy and his derivation of $\sim 10^{79}$ for the total number of elementary particles in the universe. However, assumptions employed to obtain the universal mass in a volume of $\sim 10^{79} \mathrm{~m}^{3}$ produce values remarkably commensurate with $\sim 10^{79}$ protons. The congruence between the calculated gravitational forces for rest mass photons separated by Planck's length and potassium ions separated by the distance that maintains a neuron's resting plasma membrane potential is also consistent with his assumption that universal structure is mirrored within consciousness and its extensions through sensitive instrumentation. Recent measurements of photon emissions during changes in membrane potentials and human beings engaging in visual imagery as well as the concept of entanglement suggest that careful reevaluation of Eddington's approach in cosmology, astronomy, and astrophysics may be revealing.
\end{abstract}

Keywords: Sir Arthur Eddington; Photons and Membrane Ions; Consciousness and Photon Emissions; Eddington Number

\section{INTRODUCTION}

The question of absolute and relative quantities has been a central dichotomy in the history of astronomy and astrophysics. Although some constants, such as $G, \varepsilon, \mu$, and $\hbar$ are considered more or less immutable [1], others, such as Eddington's cosmic number $\left(\sim 1.5 \cdot 10^{79}\right.$ ) for the total number $(\mathrm{N})$ of elementary particles in the universe, have been considered frivolous and even "absurdly precise" as stated by Liddle and Loveday [2]. Eddington assumed each elementary particle displayed a dual role [3]. One contributed to the structure of the universe and existed continuously while the other was an independent unit that may or may not exist. The relationship of a particle to the universe is considered to be a preexisting state with a value that is 0 (unoccupied) or 1 (occupied) state. At any given time the particle either exists or does not exist.

Recently Henry [4] suggested, as did Eddington, that nothing exists but observations. The cosmic number was based upon the essential requirement that any observation is referenced to the relationship between two particles. Because each particle can exist (1) or not exist $(0)$ there are four combinations $(0,0 ; 0,1 ; 1,0 ; 1,1)$. An observation requires a condition of 1,1 . As a result there are three modes of nonexistence. When a second observation is 
referenced, also with four modes, a total of 16 combinations occur where four are symmetrical and twelve are unsymmetrical.

Half of these unsymmetrical combinations are active and half are "dormant". However the latter can be activated when the electrical characteristics of the particle become manifested. The latter phenomenon is remarkably similar to contemporary descriptions of the Casimir effect [5] whereby energy transferred from the external field to "virtual" particles or vacuum oscillations" transforms the "virtual" particles to "real" ones but only if the boundary conditions depend upon time, that is, the boundaries are not static.

Two sets or entities are involved with an observation while two more are required for the standard or reference from which the measurement can emerge. Eddington's approach is similar to the fundamental concept found in all sciences where the phenomena that define the discipline are comprised of units $(n=1)$ or aggregates $(n>1)$ of units (fields) and processes (requiring time to be observed) for those units or aggregates [6]. With 16 combinations of 0,1 values there are $256(16 \cdot 16)$ kinds of comparison [3]. The $2^{256}$ combinations multiplied by the fine structure constant (137) results in the cosmic number. From Eddington's perspective this number would contain the threshold mass that closed the universe to an "infinite but bounded" condition.

The cosmic number was one of four dimensionless numbers derived from $\mathrm{c}$ (the velocity of light), $G$ (Newton's gravitational constant), q (the charge of an electron), $m_{e}$ (the mass of an electron), $\mathrm{m}_{\mathrm{p}}$ (the mass of the proton), and $\mathrm{h}$ (Planck's constant). The other three numbers were: $\mathrm{M}_{\mathrm{p}} / \mathrm{m}_{\mathrm{e}}$ or $1836,\left[\mathrm{c} / \sqrt{ }\left(\mathrm{q}^{2} / \mathrm{m}_{\mathrm{e}} \cdot \mathrm{r} \cdot 2 \pi \varepsilon_{0}\right)\right]$ or 137 (the fine structure constant), and 2.3 $\mathrm{x} 10^{39}$ the ratio for electrostatic (Coulomb) and gravitational forces (the force constant) between an electron and proton for a Bohr magneton. Several sets of equations relate the conspicuous square-root relationship between the cosmic number $\mathrm{N}$ and the force constant [3].

In his seminal book, The Nature of the Physical World (1928), Sir Arthur Eddington [7] argued that the substratum of everything in the universe is a cognitive construct that is determined by a "mental character". Within a traditional psychophysical framework, he stated that the world of physics is constructed by the human brain and hence the structure of the objective world is mirrored in our own consciousness. In the present paper I report and integrate the recent quantitative data and observations from astrophysics, astronomy, and neuroscience that might support or at least initiate a reevaluation of Eddington's controversial contentions. Numerical solutions were rounded to the first decimal for consistency.

\section{THE RELEVANCE OF EDDINGTON'S NUMBER}

The estimated radius $(\mathrm{R})$ and energy of the measurable (visible) universe can inferred by the relation, often designated as the constant of gravitation in general relativity,

$$
f=8 \pi \mathrm{G} / \mathrm{c}^{2}
$$

When its quantitative solution of $1.86 \cdot 10^{-26} \mathrm{~m} / \mathrm{kg}$ is multiplied by the inferred mass (M) of the universe of $2.4 \cdot 10^{52} \mathrm{~kg}$ [8], both the radius (R) and the energy can be estimated. $\mathrm{R}$ is $4.4 \cdot 10^{26}$ m.

The volume of a sphere with this radius is $36.4 \cdot 10^{79} \mathrm{~m}^{3}$. However the rate of change of this quantity is also changing over the surface of the boundary condition. The second order derivative $\left(\partial^{2} \mathrm{f} / \partial \mathrm{r}^{2}\right)$ for $4 \pi \mathrm{r}^{2}$ is $8 \pi$. When the derived volume of this sphere is divided by $8 \pi$ 
the adjusted volume is $1.5 \cdot 10^{79} \mathrm{~m}^{3}$. Two important implications follow. First, 1.5/36.4 is .04 (4\%). According to the Lambda-Cold Dark Matter (CDM) model this proportion reflects all of the matter and energy that composes the atoms and photons of the universe [35].

The second implication is that Eddington's concept of the single unit particle, such as the proton, would be supported. When rounded to the first digit, Eddington's number of $1.5 \cdot 10^{79}$ divided by $1.5 \cdot 10^{79} \mathrm{~m}^{3}$ would approach unity. If we assume the "elementary particle" is the mass of the proton $\left(1.6 \cdot 10^{-27} \mathrm{~kg}\right)$, then the universe's mass would be $\sim 2.5 \cdot 10^{52} \mathrm{~kg}$. This is convergent with the value obtained by equating the intrinsic universal pressure of $1.4 \cdot 10^{-10}$ $\mathrm{Pa}$ (from $\left.\partial \mathrm{c}^{2},\left(1.6 \cdot 10^{-27} \mathrm{~kg} / \mathrm{m}^{3}\right) \cdot\left(9 \cdot 10^{16} \mathrm{~m}^{2} / \mathrm{s}^{2}\right)\right)$ with the gravitational constant G. Employing that approach [8], the estimated mass was $2.4 \cdot 10^{52} \mathrm{~kg}$. This value is the same order of magnitude estimated from empirical observations of $10^{30} \mathrm{~kg}$ (per average star) assuming an average of $10^{11}$ stars per galaxy and $10^{11}$ galaxies within the universe.

The rest energy $E_{0}$, or inertial mass, of a system is equal to the mass $M_{0}$ which can be considered the characteristic of the gravitational field generated by the system. Energy is related to $\mathrm{G}$ and $\mathrm{M}$ according to the equation:

$$
\left(8 \pi \mathrm{M}^{2} \mathrm{G} / \mathrm{R}\right)=\mathrm{Mc}^{2}
$$

Assuming the same values for the mass of the universe and the radius derived from (1), the total energy equivalence is $2.2 \cdot 10^{69} \mathrm{~J}$ which is the same value as $\mathrm{Mc}^{2}$ for this mass.

The more classic approach is the relativity equation:

$$
\mathrm{R}_{\mu \mathrm{v}}-1 / 2 \mathrm{R}_{\mathrm{g} \mu \mathrm{v}}+\Lambda_{\mathrm{g} \mu \mathrm{v}}=8 \pi \mathrm{G} / \mathrm{c}^{4}\left[\mathrm{~T}_{\mu \mathrm{v}}\right]
$$

where $R_{\mu v}=$ the curvature tensor of Ricci, $R$ is an inference of scalar curvature, $g \mu v=a$ metric tensor, $\Lambda$ is the cosmological constant, and $\mathrm{T}_{\mu v}$ is the stress-energy tensor. If the later is set equivalent to the total energy of $2.2 \cdot 10^{69} \mathrm{~J}$, the distance $(\mathrm{m})$ is $4.4 \cdot 10^{26} \mathrm{~m}$ or $\mathrm{R}$, the value derived from (1).

In order to estimate the energy associated with the smallest units of space $(\Delta s)$ and time $(\Delta t)$ that define the four-dimensional structure of the universe, this total energy would be divided by $\Delta \mathrm{s}$ and multiplied by the cumulative numbers of $\Delta$ ts that have contributed continuously to the structure of the universe in order to be consistent with Eddington's assumptions. The numbers of Planck's volumes (assuming a sphere) of $4.3 \cdot 10^{-105} \mathrm{~m}^{3} / \mathrm{PLV}$ (Planck's length voxel) within the $1.5 \cdot 10^{79} \mathrm{~m}^{3}$ adjusted volume is $0.3 \cdot 10^{184} \mathrm{PLV}$ and each voxel would be equivalent to $6.3 \cdot 10^{-115} \mathrm{~J}$. Assuming 13.3 billions years as the age of the universe a total of $0.8 \cdot 10^{61}$ PT (Planck's time) increments have elapsed resulting in a cumulative energy of $\sim 5 \cdot 10^{-54} \mathrm{~J}$.

This suggests that the rest mass, $\mathrm{m}_{\gamma}$, of a photon where $\mathrm{c}^{2} \rightarrow \mathrm{c}=1$ would be $5 \cdot 10^{-54} \mathrm{~kg}$ which is within $8 \pi$ of the $<1 \cdot 10^{-52} \mathrm{~kg}$. This quantity is the currently accepted upper limit for the rest mass of a photon [9]. The likelihood that an experiment could demonstrate that photon has a rest mass that is exactly zero is very small. An inferred value for the upper limit of $\mathrm{m}_{\gamma}$ from satellite measurements in the geomagnetic field was $<1 \cdot 10^{-51} \mathrm{~kg}$. The estimate from Jupiter's magnetic field was $<8 \cdot 10^{-52} \mathrm{~kg}$.

The convergence to unity of $\mathrm{c}^{2}$ to $\mathrm{c}$ near Planck's boundaries has quantitative support. There are in the order of $10^{61}$ Planck's lengths within the radius of the universe and $10^{61}$ Planck's times resulting in a $\Delta \mathrm{s} / \Delta \mathrm{t}$ ratio approaching 1 . Consequently, the same parameters that are congruent with Eddington's cosmic number predict the upper limit of the rest mass of 
the photon that is similar in magnitude to those estimates derived from more complex mathematical assumptions.

Implications of a non-zero rest mass for a photon have been delineated superbly by $\mathrm{Tu}$ et al [9] and are applicable to Eddington's philosophy. They include: 1) the emergence of a frequency dependence in the velocity of the propagation of electromagnetic waves through free space, 2) the emergence of a third state of polarization or a "longitudinal" photon, 3) a reformulation of the velocity of light that is not a unique constant but a function of frequency, and 4) the possibility that the Aharonov-Bohm phase shift for electrons around a tube of magnetic flux due to the presence of the " $A$ " vector potential are potential "non-local" effects.

These conditions could contribute to the type of photon entanglement that would qualify for a process that allows two of these "entities" to be related such that a measurement of one photon now could affect the polarity of the other photon in the pair when they are separated by billions of years.

\section{NEUROPHYSICAL EVIDENCE FOR EDDINGTON'S ASSUMPTIONS}

The relationship between gravity and electromagnetism (EM) has been a major challenge to both theoretical physics and astrophysics. If Eddington were alive today he might argue the solution is quantitative and not qualitative and there may not be a "grand equation" in the form anticipated by most scientists. The central operating relationships for all forces is the product of the quantity divided by the square of the distance multiplied by a force-specific constant, that is

$$
\left[\left(\mathrm{m}_{1} \cdot \mathrm{m}_{2}\right) /\left(\mathrm{d}_{1} \cdot \mathrm{d}_{2}\right)\right] \cdot \mathrm{k}
$$

Eddington's epistemology argues that the structure of the objective world is precisely represented within the consciousness of the observer such that a quantitative convergence of discrete values between essential properties of the universe and the conditions of the human brain associated with consciousness would be required.

If we applied the formula for gravity $(G)$ to the essential unit of EM, the photon, then the product of the rest mass [9] of two photons, divided by the square of the smallest distance (Planck's length), would be $\left(6.67 \cdot 10^{-11} \mathrm{~m}^{3} / \mathrm{kg} \mathrm{s}^{2}\right) \cdot\left(10^{-104} \mathrm{~kg}^{2}\right) \cdot\left(1.62 \cdot 10^{-35} \mathrm{~m}\right)^{-2}$ or $\sim 5 \cdot 10^{-45}$ $\mathrm{N}$. This is an extremely small force. When spread over the estimated length (width) of the universe $\left(\sim 10^{26} \mathrm{~m}\right)$ the energy values would be in the order of $10^{-20}$ to $10^{-19} \mathrm{~J}$, depending upon assumptions of universal age.

If the more specific value obtained directly from the Eddington Number for the upper limit of the rest mass $\left(5 \cdot 10^{-54} \mathrm{~kg}\right)$ is employed the force is $6.4 \cdot 10^{-48} \mathrm{~N}$ and when applied to the circumference defined by $\mathrm{R}\left(4.4 \cdot 10^{26} \mathrm{~m}\right)$ the energy is $1.8 \cdot 10^{-20} \mathrm{~J}$. As predicted by Eddington's psychophysical approach, this magnitude of energy $\left(\sim 2 \cdot 10^{-20} \mathrm{~J}\right)$ has been recently considered to be an essential neuroquantum for phenomena involved with transmission of information within brain space through both chemical and electromagnetic forms [10].

From Eddington's perspective [7], particularly his approach that the world of physics is constructed from messages transmitted along nerves to the seat of consciousness, these values $\left(\sim 2 \cdot 10^{-20} \mathrm{~J}\right)$ would be relevant because they are also the energies associated with the effect of the change of $\sim 1.2 \cdot 10^{-1} \mathrm{~V}(\Delta \mathrm{v}=-90$ to $+30 \mathrm{mV})$ during an action potential of a neuron upon a 
unit charge $\left(1.6 \cdot 10^{-19} \mathrm{~A} \cdot \mathrm{s}\right)$ as well as the energy required to stack one base nucleotide on a ribbon of RNA [10].

The former, action potentials, are the essential units of thought and awareness while the RNA sequences are the initial physical substrate for the "representation of experience", that is memory, in patterns of proteins expressed as spines on dendrites of neurons within the cerebral volume.

Calculations [10] based upon the concentrations of potassium ions required to maintain the resting (voltage) potential of the neuronal plasma membrane indicate that only about $2 \cdot 10^{6}$ ions (assuming the average neuron with a diameter of $10 \mu \mathrm{m}$, this number of ions is the product of the surface area of $\pi \cdot 10^{-6} \mathrm{~cm}^{2}, 1 \mu \mathrm{F} / \mathrm{cm}^{2}$ capacitance and $-90 \mathrm{mV}$ resting potential, divided by the unit charge $1.61 \cdot 10^{-19} \mathrm{~A} \cdot \mathrm{s}$ ) within a thin shell around the membrane are involved.

The distance between each of these numbers of ions over the surface of the membrane would be $\sim 10 \mathrm{~nm}$. With this average distance the electric force would be $\sim 2 \cdot 10^{-12} \mathrm{~N}$ resulting in energies of $\sim 2 \cdot 10^{-20} \mathrm{~J}$ between the ions on this surface. Effectively the energy of the action potential is the transformation in discrete time $(1 \mathrm{msec})$ of the energy associated with the resting membrane potential. Decades earlier Bohr [11] had suggested that such small increments of energy at quantum levels might determine the essential features of thinking and hence there was a possibility that thought and matter could be mutually interactive.

Eddington's insight is revealed when the force of gravitational attraction between two (separated by $\sim 10 \mathrm{~nm}$ ) potassium ions, the primary physical substrate for the membrane polarization required for consciousness, is calculated. This value is $\sim 10^{-45} \mathrm{~N}$. This is the same order of magnitude as the gravitational attraction between the rest mass $\left(10^{-52} \mathrm{~kg}\right)$ of photons separated by Planck's distance that may be coupled to the emergence of electromagnetic quantum within the wavelengths of visible light and near infrared radiation [12]. To obtain the value of $10^{-20} \mathrm{~J}$, the range of the essential neuroquantum through which the physical correlates of consciousness appear to operate $[10,13]$, this force must be distributed over a distance of about $10^{26} \mathrm{~m}$, well within the range of the estimated width of the universe.

This convergence might be considered a potential support for Eddington's contention that the objective world is precisely "mirrored" within our own consciousness [7]. Eddington pursued the possibility that understanding the relationship between the fine structure constant, the ratio of mass between protons and electrons, and the total numbers of protons in the universe, might reveal some recondite reflection of the "cognitive construct". No doubt he would have appreciated Gödel's incompleteness theorem (or proof) that indicates within any complex logical system there is always one axiom, the reference, which cannot be refuted or even discerned within that system. In this context the system would be the cerebral processes by which the human brain engages in problem solving regarding the boundaries of the total set (the space-time parameters of the "universe").

With an estimated mass of the universe in the order of $10^{52} \mathrm{~kg}[8]$ and the upper rest mass of a photon of $10^{-52} \mathrm{~kg}$ [9], the total numbers of rest-mass photons would be $10^{104}$.

This value is an identity with the solution required for the four dimensional tensor $\left(10^{104}\right.$ $\mathrm{m}^{4}$ ) required to satisfy the equivalence between $G$ and intrinsic pressure obtained from the product of proton density and the square of the velocity of light [8]. Assuming a universal volume $10^{79} \mathrm{~m}^{3}$, there would be an average of $10^{25}$ potential rest-mass photons per $\mathrm{m}^{3}$ or about one per volume with a diameter that is within the range of the neuronal plasma membrane $(10 \mathrm{~nm})$ and its ion channels $(1 \mathrm{~nm})$. This does not prove Eddington's psychophysical model but does open alternative appreciations for the relationship between cosmological observations and phenomena that occur at the spatial level of the brain cell 
membrane and the conduits (the "channels") by which its vitality and processes are maintained.

One possible connection between gravitational and electromagnetic phenomena that would satisfy Eddington's approach for quantitative unity would be the numbers of photons within a thin section overlapping with visible wavelengths $\left(10^{14}\right.$ to $10^{15} \mathrm{~Hz}$, i.e., 300 to 3000 $\mathrm{nm}$ ) of the numbers of rest-mass photons per unit volume [12]. A median number would be between $10^{18}$ and $10^{19}$ photons $/ \mathrm{m}^{2}$. If the rest mass $\left(10^{-52} \mathrm{~kg}\right)$ of the photon is multiplied by $\mathrm{c}$ $\left(3 \cdot 10^{8} \mathrm{~m} / \mathrm{s}\right)$ and the frequency range of $10^{14} \mathrm{~Hz}$ to $10^{15} \mathrm{~Hz}$ the result is between $10^{-29}$ to $10^{-30}$ $\mathrm{N}$. With a cross-sectional density of $\sim 10^{19}$ photons $/ \mathrm{m}^{2}$, the "photons" would produce pressures between $10^{-11} \mathrm{~Pa}$ and $10^{-10} \mathrm{~Pa}$.

This is within the same range as the value [8] employed to estimate the mass of the universe and the order of magnitude that could be considered consistent with Eddington's Number.

\section{NEUROQUANTUM OF THOUGHT, BIOPHOTON EMISSIONS AND ENTANGLEMENT}

Developments within quantal neurophysics strongly support Eddington's philosophical idealism. Although the emission of light (biophotons) from biological systems had been reported in his life time [14] their implications were not apparent to the contemporary thinking. Cells and aggregates of cells emit photons in the order of $10^{6}$ photons $/ \mathrm{m}^{2} \cdot \mathrm{s}[15]$ due to a variety of biochemical pathways [16]. Recently Dotta et al [17,18] experimentally demonstrated that the biophoton emissions originate from the cellular plasma membrane at incremental quanta in the order of $10^{-20} \mathrm{~J}$ and this value is directly coupled to the resting membrane potential. Such single discrete increments of energy are relevant to perception and to consciousness.

There are several recent experimental demonstrations that the action potential of only one neuron can affect the activity of an overt complex behavior in mammals [19] and the state-dependent organization of the entire cerebral manifold [20].

Bokkon [21] hypothesized that human imagery, or thought, was associated with biophoton emissions. Within the last two years direct measurements with photomultiplier tubes have verified that imagining white light, for example, is associated with reliable increases in photon emissions from (particularly) the right hemisphere of the human brain. Both dreaming and the influences from subtle changes in geomagnetic activity exhibit a more right than left hemispheric component. The average increases in photon emissions are about $5 \cdot 10^{-11} \mathrm{~W} / \mathrm{m}^{2}$, or when the medial cross-sectional area of the human cerebrum is considered, about $5 \cdot 10^{-12} \mathrm{~J} / \mathrm{s}[22,23]$. With $10^{-20} \mathrm{~J}$ associated with each action potential, the photon emission associated with "visual imagery" would involve 10 to 100 million neurons each firing at about $10 \mathrm{~Hz}$, values that are consistent with direct inferences from functional Magnetic Resonance Imaging (fMRI).

What may have delighted Eddington and would be predicted by his approach is the energy density of the photon output $\left(\sim 10^{-10} \mathrm{~W} / \mathrm{m}^{2}\right)$ from the brain during ideational perception when applied to the level of the neuronal membrane. The increment of energy within the cross-sectional area $\left(10^{-16} \mathrm{~m}^{2}\right)$ for a $10 \mathrm{~nm}$ plasma membrane or the separation of the ions on the membrane's surfaces that maintains its potential would be in the order $10^{-26} \mathrm{~W}$. A background of about a unit Jansky $\left(10^{-26} \mathrm{~W} / \mathrm{m}^{2} \cdot \mathrm{Hz}\right)$ is routinely measured from the most distant galaxies [24]. 
From the perspective of some modern models of brain and consciousness $[25,26]$, the difference in surface area associated with this level of energy is not necessarily a limit. The total surface area of all of the membranes of single neuronal somas $\left(\sim \pi \cdot 10^{-10} \mathrm{~m}^{2}\right)$ multiplied by the total numbers of cerebral cortical neurons $\left(2 \cdot 10^{10}\right)$ in one hemisphere is in the order of $1 \mathrm{~m}^{2}$. Such congruence satisfies one condition of a classic hologram [26]. Again, Eddington's contention of the convergence of numerical properties for the universe and neuronal (consciousness) activity would be supported.

The emission of thinking-neuronal, membrane-coupled photons is specifically relevant to Eddington's concept when one considers that the time required for a photon at velocity c to traverse a $10 \mathrm{~nm}$ membrane is $\sim 10^{-16} \mathrm{~s}$ which is the approximate time required for one orbit of an electron around the Bohr magneton. That coupling between photons, orbital durations of electrons, and membrane activity can occur has also been shown experimentally. If cells or human brains are separated by significant distances and both share the same electromagnetic field configuration with specific shifts in circular angular acceleration, light stimulation of one is excessively correlated ("entangled") with increased photon emissions from the other [27]. In other words, when light was presented to cells or brains in one location light emission with densities expected for membrane processes occurred from the cells or brains in the second location.

Eddington's epistemology that assumes a mutual reflection between properties of consciousness and the presumed properties of the universe anticipated the concept of influence of intention and entanglement [13]. The possibility that thought (observation) could affect the universe in terms of altering bifurcation of processes or its rate of change has been considered seriously. In the enigmatic domain of quantum philosophy [28] where the observation or measurement of a photon from a distant star simultaneously changes the polarity of the paired photon eons before the present, philosophical idealism is a necessary condition.

\section{THE ELECTROMAGNETIC FIELD STRENGTHS OF CONSCIOUSNESS AND INTRAGALACTIC SPACE}

Eddington's contention that the physical world is mirrored in consciousness now has empirical support. The strengths of cerebral magnetic fields associated with consciousness are in the order of $10^{-11} \mathrm{~T}$ to $10^{-12} \mathrm{~T}(\mathrm{pT})$. These calculated values emerge at both the level of the individual ion channel as well as the entire cerebral manifold. Recent measurements or observations have revealed magnetic field strengths in the order of $10^{-10} \mathrm{~T}$ within galaxies and unamplified values with upper limits in the $10^{-13} \mathrm{~T}$ range for extragalactic fields. Experimental laboratory research indicates that minute changes in electron-proton phenomena associated with applications of weak applied magnetic fields involving specific parameters for changing angular velocity [18] may facilitate entanglement as described by $\mathrm{Wu}$ and $\mathrm{Hu}$ [28].

The induced magnetic moment [29] corresponding to a change in angular velocity (opposite to the applied field) can be described as:

$$
\Delta \mathrm{m}=\left[e^{2} \cdot \mathrm{r}^{2} / 4 \mathrm{~m}_{\mathrm{e}}\right] \cdot \mathrm{B}
$$

where $\mathrm{r}$ is the Bohr radius of $5.3 \cdot 10^{-11} \mathrm{~m}$. 
The application of a $10 \mathrm{pT}$ field results in a magnetic moment of $1.8 \cdot 10^{-40} \mathrm{~A} \cdot \mathrm{m}^{2}$ or $\mathrm{J} / \mathrm{T}$. If this field is immersed within a second $10^{-11} \mathrm{~T}$ pervasive magnetic field, such as those measured within galaxies, then the total energy is around $1.8 \cdot 10^{-51} \mathrm{~J}$.

This energy is remarkably similar to the energy per Planck's voxel over the summed Planck's times since the beginning of the universe. Assuming $c^{2} \rightarrow c$, this unity would be the upper limit of the rest mass of a photon. Even this small energy becomes relevant when the period is discerned by dividing Planck's constant, $6.4 \cdot 10^{-34} \mathrm{~J} \cdot \mathrm{s}$, by $1.810^{-51} \mathrm{~J}$. The "duration" (one "cycle") of this energy would be $\sim 3.7 \cdot 10^{17}$ s or approximately 12 billion years.

\section{EDDINGTON'S CONCEPT OF CONSCIOUSNESS AND GRAVITY}

Sakharov's model as presented by Puthoff [30] assumes that gravity, rather than being a separately existing force, is an induced effect associated with zero-point fluctuations of the vacuum when matter is present. Calculations by Ahmed et al [31] indicated that the effects of gravity (as inferred by weightlessness) on the human electroencephalographic activity (EEG), assuming an axon conduction of $\sim 20 \mathrm{~m} / \mathrm{s}$, was about 2 parts per million. EEG activity, the time-varying potential differences measured between two discrete points on the skull's surface, is an emergent property of averaged action potentials from millions of neurons within the cerebral cortices.

The bulk velocity associated with the cohesive cerebral waves most strongly associated with consciousness is $\sim 4 \mathrm{~m} / \mathrm{s}$ [25]. These parameters determine a fundamental $\sim 7$ to $8 \mathrm{~Hz}$ resonance, that is

$$
\mathrm{f}=[\mathrm{n}(\mathrm{n}+1)]^{1 / 2} \cdot \mathrm{v} / 2 \pi \mathrm{r}
$$

with an average cerebral circumference of $\sim 55 \mathrm{~cm}$.

Eddington [7] would very likely have considered that if the quantum of electromagnetic energy the brain perceives as light is a subset of the constant condition of gravitational attraction between every photon at its rest mass across the universe, a special condition could exist where $\mathrm{c}^{2} \rightarrow \mathrm{c}$, that is the limit for an identity or "singularity". In this instance the energy would be "equal" to the mass $\left(10^{-52} \mathrm{~kg}\right)$. As a result, the electromagnetic energy of $10^{-52} \mathrm{~J}$ when divided by the duration $\left(\sim 10^{-44} \mathrm{~s}\right)$, for one Zitterbewegung frequency, is about $10^{-8} \mathrm{~J} / \mathrm{s}$.

The proportion of that energy associated with one orbit of an electron $\left(10^{-16} \mathrm{~s}\right)$ for the Bohr magneton would be $\sim 10^{-24} \mathrm{~J}$. The frequency equivalence for this quantum of energy is remarkably approaching the precession frequency of neutral hydrogen $(1.42 \mathrm{GHz})$ which composes most of the matter of the universe [36-38]

If gravity affected cerebral activity by the proportion suggested by Ahmed et al (2 parts per 10 million, [31]), then one complete phase shift between two peaks would be: $2 \cdot 10^{7}$ divided by $1.42 \cdot 10^{9} \mathrm{~Hz}$, or about $14 \mathrm{msec}$. This value is precisely within the range measured empirically [32] as the phase-shift durations associated with human consciousness as well as with the "re-entrant" process or rates of change associated with conscious awareness [33]. 


\section{EDDINGTON'S NUMBER, THE AGE OF THE UNIVERSE, AND HIS "NON-EXISTENT" STATES}

There are still unresolved issues concerning the structure of the space (between $10^{-35} \mathrm{~m}$ and $10^{-16} \mathrm{~m}$ ) within which matter and energy exists. The problem occurs because $\Delta \mathrm{s}$ (an observation of space) and $\Delta \mathrm{t}$ (the time required to discern this space) appear to be related quantitatively [6]. A picosecond is optimal to discern phenomena at the level of a picometer (an atom), a millisecond is optimal to discern phenomena at the level of a mm (the action potential of an axon), and a megasecond is optimal to discern phenomena at the level of a megameter (geophysical events). In order to observe or measure the process at least two $\Delta$ ts are required, the Nyquist limit. However at the level of the entire universe where there is only $1 \Delta \mathrm{s}$ and hence $1 \Delta \mathrm{t}$, there is no "time" or "change". At this level unusual relationships that reflect the largest and smallest measurements also converge [34].

It may not be coincidence that to contain the volume of a "spherical" electron (from the classic radius) of $9.4 \cdot 10^{-44} \mathrm{~m}^{3}$ in a "string" with a cross sectional area of Planck's length $\left(2.1 \cdot 10^{-70} \mathrm{~m}^{2}\right)$ would require a length of $\sim 4.5 \cdot 10^{26} \mathrm{~m}$, the $\mathrm{R}$ derived from gravitational energy (1) that resulted in the accommodated volume which reflected Eddington's Number. Assuming $\mathrm{c}$ has not changed a radius of that value from a central source, would suggest the age of the universe is more approximate to 47.6 billions of years. Consequently the current estimated age is about one-quarter of the value (the proportion of existence) predicted by the horizon that facilitates Eddington's number. The remaining proportion could indicate the three modes of non-existence.

A second estimation of the "alternative" age of the universe is derived from Go. Assuming 1 proton $/ \mathrm{m}^{3}$ the value would be $0.3 \cdot 10^{19} \mathrm{~s}$ or 95 billions of years (twice 47.6 billons of years). If the 95 billions of years reflect the total set of possibilities, then only $\sim 14 \%$ of the matter and energy has occurred while the remaining "states", to employ Eddington's concept, have yet to occur. If Eddington were assessing the dominant proportions of "dark energy" and "dark matter" in contemporary cosmology, would he have stated that the remaining $86 \%$ is matter and energy that has not yet occurred? In other words if there is potential energy there is also potential matter? This would be consistent with his assumption that particles display duality in which they exist continuously to contribute to the structure of the universe while the other condition is a value that may or may not exist at any given time.

\section{CONCLUSION}

The final reference of measurements for all astronomical and astrophysical phenomena is the human observer, specifically the cerebral activity associated with perception, thought, and imagination. Our most sophisticated instruments have been constructed to be compatible with these intrinsic constraints of biological activity.

The range in explanations for the manner by which we perceive and measure the universe through these sensitive instrumental extensions of perceptual processes have ranged between materialism (reality is determined by matter) to idealism (reality is determined by thought). Eddington's emphasis along the latter portion of the continuum during his life time at that stage in the evolution of astrophysical concepts was precocious and certainly courageous. These two approaches are present today in the concepts of mass (particles) vs energy (waves), that he described as "wavicles", and in the highly recalcitrant adherence to mind-body dualism. However, the energies associated with the processes of thought and the 
fundamental characteristics of matter, as predicted by Bohr [11], may exist within the same order of magnitude, may be capable of interaction, may extend throughout the universe [29], and may have been present indefinitely [28].

For example the numbers of neurons within a cerebral cortical column $(\sim 0.5$ to $1 \mathrm{~mm}$ in width by 3 to $5 \mathrm{~mm}$ thickness), the functional module of human brain function, is about $15 \cdot 10^{4}$. With each neuron discharging at $\sim 10 \mathrm{~Hz}$ and each action potential $\sim 10^{-20} \mathrm{~J}$ the energy is about $10^{-14} \mathrm{~J}$ which is equivalent to a frequency $10^{19} \mathrm{~Hz}$ or a wave length remarkably approaching the diameter of the Bohr magneton when coefficients are included. The magnetic moment of a Bohr magneton, $\sim 9 \cdot 10^{-24} \mathrm{~J} / \mathrm{T}$, for the intensities of the magnetic field component associated with consciousness $(\sim 1 \mathrm{pT})$ is $10^{-36} \mathrm{~J}$ which is the equivalent $\left(\mathrm{mc}^{2}\right)$ of the upper limit of the rest mass of a photon, $10^{-52} \mathrm{~kg}[9,29]$.

Considering the persistence of ephemeral anomalies, intermittent inconsistencies, and cognitive conundrum that have been observed or inferred in complex databases for astronomical phenomena, Eddington's considerations may be very relevant. Endorsement of some of his insights, such as his model for radiative energy transport for stars, the balance between gravitational attraction and radiation pressure, and the Eddington-Weinberg relation, but the exclusion of the philosophical bases upon which his discoveries were based (as well as his less popular derivations such as his number) may have produced a conceptual scotoma. Eddington grasped the significance of relativity theory long before most others. There is no $a$ priori reason to assume that his perspicacity ended there.

\section{ACKNOWLEDGMENTS}

This manuscript is dedicated to the memory and genius of Sir Arthur Stanley Eddington and to his prescience of concepts yet to be developed. Thanks to Stanley A. Koren for his tireless dedication to shadow calculating my computations on Friday afternoons.

\section{References}

[1] V. N. Mansfield and S. Malin, Astrophysical Journal 209 (1976) 335-349.

[2] A. Liddle and J. Lovejoy, Companion to Cosmology, Oxford University Press 2008, p.109.

[3] J. Singh, Great ideas and theories of modern cosmology, Dover Press 1961.

[4] R. C. Henry, Nature 436 (2005) 29.

[5] M. Bordag, U. Mohideen, V. M. Mostepanenko, Physics Reports 353 (2001) 1-205.

[6] M. A. Persinger, Perceptual and Motor Skills 88 (1999) 1351-1355.

[7] A. Eddington, "Nature of the physical world" U. Michigan Press 1981.

[8] M. A. Persinger, Journal of Physics, Astrophysics and Physical Cosmology 3 (2009) 1-4.

[9] L. C. Tuo, J. Luo, G. T. Gilles, Progress in Physics 68 (2009) 77-130.

[10] M. A. Persinger, Current Medicinal Chemistry 17 (2010) 3094-3098.

[11] N. Bohr, Atomic Physics and Human Knowledge, Wiley and Sons 1958. 
[12] M. A. Persinger, The Open Astronomy Journal 5 (2012) 41-43.

[13] M. A. Persinger, S. A. Koren, G. F. Lafreniere, Neuroquantology 6 (2008) 262-271.

[14] A. A. Gurwitch, Experientia 44 (1988) 545-550.

[15] F. A. Popp, "Coherent photon storage of biological systems", in F. A. Popp, G. Becker, H. L. Konig, W. Pescha, eds, Electromagnetic Information. Urban and Schwarzenberg, Munchen-Wein-Baltimore 197).

[16] Y. Katoka, A. Cui, M. Yamagata, T. Niigagaki, Y. Oshi, Biochemical and Biophysical Research Communications 285 (2011) 1007-1011.

[17] B. L. Dotta, C. A. Buckner, D. Cameron, R. Lafrenie, M. A. Persinger, General Physiology and Biophysics 30 (2011) 301-309.

[18] B. L. Dotta, M. A. Persinger, Journal of Biophysical Chemistry 3 (2012) 72-80.

[19] R. R. Houweling, M. Brecht, Nature 451 (2008) 65-69.

[20] L. T. Yu-Cheng, P. Mu-ming, D. Yang, Science 324 (2009) 643-646.

[21] I. Bokkon, Sleep and Hypnosis 7 (2005) 61-76.

[22] B. T. Dotta, M. A. Persinger, Journal of Consciousness Exploration \& Research 2 (2011) 1463-1473.

[23] B. T. Dotta, K. S. Saroka, M. A. Persinger Neuroscience Letters 513 (2012) 151-154.

[24] S. A. Koren, M. A. Persinger, Journal of Physics, Astrophysics and Physical Cosmology 4 (2010) 1-4.

[25] M. A. Persinger, C. F. Lavallee, Journal of Consciousness Exploration and Research 1 (2010) 785-807.

[26] M. A. Persinger,C. F. Lavallee, Journal of Consciousness Studies 19 (2012) 128-153.

[27] B. T. Dotta, C. A. Buckner, R. M. Lafrenie, M. A. Persinger, Brain Research 388 (2011) 77-88.

[28] H. Hu, M. Wu, Neuroquantology 1 (2006) 5-16.

[29] M. A. Persinger, Journal of Cosmology 14 (2010) 113-119.

[30] H. E. Puthoff, Physical Review A 39 (1989) 2333-2342.

[31] S. N. Ahmed, S. A. Kamal, K. A. Siddiquui, S. A. Husain, M. Naeem, Kar University Journal of Sciences 25 (1997) 19-24.

[32] R. R. Llinas, D. Pare, Neuroscience 44 (1991) 521-535.

[33] G. M. Edelman, The remembered present: a biological theory of consciousness, Basic Books 1989.

[34] M. A. Persinger, S. A. Koren, International Journal of Neuroscience 117 (2007) 157-175.

[35] Tomasz Borowski, International Letters of Chemistry, Physics and Astronomy 1 (2012) 1-5. 
[36] Zdzisław Pluta, Tadeusz Hryniewicz, International Letters of Chemistry, Physics and Astronomy 4 (2012) 8-16.

[37] Zdzisław Pluta, Tadeusz Hryniewicz, International Letters of Chemistry, Physics and Astronomy 5 (2012) 35-45.

[38] Mukul Chandra Das, Rampada Misra, International Letters of Chemistry, Physics and Astronomy 7(2) (2013) 73-84. 